\title{
Cataract Surgery in Malignant Glaucoma from Complete Subluxation of Lens into Anterior Chamber
}

\author{
Soshian Sarrafpour ${ }^{1}$, Isaiah Davies ${ }^{2}$, Osama Ahmed ${ }^{3}$, Ji Liư ${ }^{4}$, Christopher C Teng $^{5}$
}

\begin{abstract}
Aim and objective: To report an unusual and difficult case of malignant glaucoma induced by a traumatic mature cataract that had migrated into the anterior chamber in a developmentally delayed patient.

Background: Malignant glaucoma can present due to multiple mechanisms, including trauma-induced cataract migration into the anterior chamber.

Case description: A developmentally delayed female in her 40s with a history of self-abuse was found to have malignant glaucoma in the setting of a traumatic cataract that had migrated in front of the iris into the anterior chamber. Exam under anesthesia and ultrasound biomicroscopy (UBM) demonstrated complete subluxation of the lens into the anterior chamber. Perioperative techniques included prophylactic pars plana vitrectomy to alleviate posterior pressure as well as the creation of an irido-zonulo-hyaloido-vitrectomy (IZHV), which allowed for reformation of the anterior chamber. These maneuvers allowed for cataract surgery to be performed through a clear corneal incision, with anterior chamber intraocular lens implantation. The patient had improved vision, pressure, and pain.

Conclusion: Malignant glaucoma can present in unique ways and the presence of a flat anterior chamber and increased posterior pressure can make surgery challenging. Initial pars plana vitrectomy in addition to the creation of an IZHV can help relieve posterior pressure and facilitate cataract extraction. It is important to factor in patient-specific situations and goals while selecting an intraocular lens.

Clinical significance: Traumatic cataracts and malignant glaucoma can present in unique scenarios that may prove difficult to treat. Certain techniques can facilitate these challenging surgeries and provide the optimal outcome for patients.

Keywords: Anterior chamber lens subluxation, Case report, Irido-zonulo-hyaloido-vitrectomy, Malignant glaucoma, Surgical management, Traumatic cataract.

Journal of Current Glaucoma Practice (2021): 10.5005/jp-journals-10078-1320
\end{abstract}

\section{BACKGROUND}

An angle-closure glaucoma is a group of glaucoma diseases associated with appositional or synechial closure of the anterior chamber angle. Multiple mechanisms can cause angle-closure including pupillary block, lens-related events, and malignant glaucoma. Angle-closure may also be related to iris and choroidal volume shifts and changes. ${ }^{1}$ In trauma-related cases, zonules may become damaged and the natural lens can shift forward, which may induce malignant glaucoma.

Cataract surgery related to trauma is commonly encountered and presents its own unique challenges. A study by the World Health Organization estimated approximately 55 million eye injuries per year worldwide with up to 1.6 million people losing vision as a result. ${ }^{2}$ Complications related to traumatic cataracts can include zonular instability, capsular fibrosis, capsular rupture, and limited visualization. Fortunately, many techniques and devices have been developed to reduce complications and maximize visual outcomes in these cases including capsular stains to improve visualization, capsular stabilization devices, and the use of anterior vitrector to open fibrotic capsules.

Rarely, trauma can result in anterior subluxation of the crystalline lens. This can also occur in association with connective tissue disorders such as Marfan syndrome or Ehlers-Danlos syndrome, exfoliation syndrome, homocystinuria, etc. It has also been reported to occur spontaneously in patients without any known risk factors. ${ }^{3}$ Anterior subluxation can be an ophthalmological emergency, with complications such as acute angle-closure crisis due to pupillary block and corneal endothelial damage secondary to lens-corneal
1,4,5 Department of Ophthalmology and Visual Science, Yale School of Medicine, New Haven, Connecticut, USA

${ }^{2,3}$ Department of Ophthalmology, Yale University, New Haven, Connecticut, USA

Corresponding Author: Soshian Sarrafpour, Department of Ophthalmology and Visual Science, Yale School of Medicine, New Haven, Connecticut, USA, e-mail: soshian.sarrafpour@yale.edu

How to cite this article: Sarrafpour S, Davies I, Ahmed O, et al. Cataract Surgery in Malignant Glaucoma from Complete Subluxation of Lens into Anterior Chamber. J Curr Glaucoma Pract 2021;15(3):164-167.

Source of support: Nil

Conflict of interest: None

touch. ${ }^{4}$ Elevated pressures and prolonged angle closure can create synechiae, leading to chronic angle closure. Another possible complication of anterior lens subluxation is malignant glaucoma. ${ }^{5,6}$ Management is diverse and individualized, including medical options such as intraocular pressure (IOP) lowering medications and cycloplegia, which allows for the lens to return to the posterior chamber. Surgical management includes cataract extraction by way of extracapsular, intracapsular, or posterior approach with vitrectomy and irido-zonulo-hyaloido-vitrectomy (IZHV), to create a one chamber environment to relieve posterior pressure., ${ }^{4,10}$

We report a unique case that required prophylactic pars plana vitrectomy decompression, IZHV, and complex cataract surgery in a patient with malignant glaucoma induced by complete subluxation

() The Author(s). 2021 Open Access This article is distributed under the terms of the Creative Commons Attribution 4.0 International License (https://creativecommons. org/licenses/by-nc/4.0/), which permits unrestricted use, distribution, and non-commercial reproduction in any medium, provided you give appropriate credit to the original author(s) and the source, provide a link to the Creative Commons license, and indicate if changes were made. The Creative Commons Public Domain Dedication waiver (http://creativecommons.org/publicdomain/zero/1.0/) applies to the data made available in this article, unless otherwise stated. 
of a mature cataract into the anterior chamber due to self-induced trauma.

\section{Case Description}

A developmentally delayed woman in her 40 s presented with rightsided ocular pain and injection. Based on her caretaker's report, she had a red right eye for 2 months duration before the presentation. Notably, she had an extensive history of self-induced harm including hitting herself on the head and poking the right eye. The initial examination was limited by her cooperation. Visual acuity was no response to bright light in the right eye and fixes and follows in the left eye. There was a moderate right eye afferent pupillary defect. The right eye was very firm on palpation with $2+$ injection and was found to have a mature white cataract and shallow anterior chamber. The left eye was unremarkable and soft to palpation. She was diagnosed with malignant glaucoma in the setting of anterior subluxation of the lens likely from trauma-related zonular loss. She was started on topical medications and oral acetazolamide. Due to a limited exam in the office, an examination under anesthesia was planned with concomitant cataract extraction. On examination under anesthesia, there was a flat anterior chamber and a 4+ mature cataract located in the anterior chamber (Figs $1 \mathrm{~A}$ and $\mathrm{B}$ ). Intraocular pressure remained elevated in the $40 \mathrm{~s}$. The left eye anterior segment examination was unremarkable. Gonioscopy confirmed a flat anterior chamber, with the lens in the anterior chamber and no visible angle structures in the right eye (Fig. 1C) and open angles in the left eye. Ultrasound biomicroscopy (UBM) was performed and confirmed the above findings (Fig. 2). Dilated fundus examination of the right eye was limited by the cataract. The left eye revealed 0.2 cup-to-disk and mild mottling of the macula. B-scan ultrasound revealed a flat retina with no choroidal effusion or retinal detachment in the right eye (Fig. 3). Axial lengths were right eye $22.95 \mathrm{~mm}$ and left eye $22.45 \mathrm{~mm}$. Keratometry was right eye $42.75 \times 163,46.25 \times 73$ and left eye $42.75 \times 7,44.0 \times 97$. The intraocular lens measurements were calculated.

Following examination under anesthesia, the right eye was prepped and draped in the usual sterile fashion. Pars plana decompression with a $25 \mathrm{G}$ vitrector was performed until the eye was palpated to be soft. A paracentesis was created, and viscoelastic material was injected into the anterior chamber with the only mild formation of the anterior chamber. The decision was made to create an IZHV to create a one chamber eye via a pars plana approach. Once this was performed, there was a noticeable decrease in IOP based on palpation and the anterior chamber was able to be deepened using viscoelastic. A dense fibrotic anterior capsule was noted; therefore, the vitrector was used to create a capsulotomy. Gentle hydrodissection was performed, followed by phacoemulsification of the lens in the anterior chamber. After lens nuclear and cortical removal, an intact capsular bag remained and was confirmed to be anterior to the iris.

Due to extensive zonular loss, the decision was made to remove the capsule with the vitrector and place an anterior chamber intraocular lens (ACIOL). The limbus-to-limbus distance
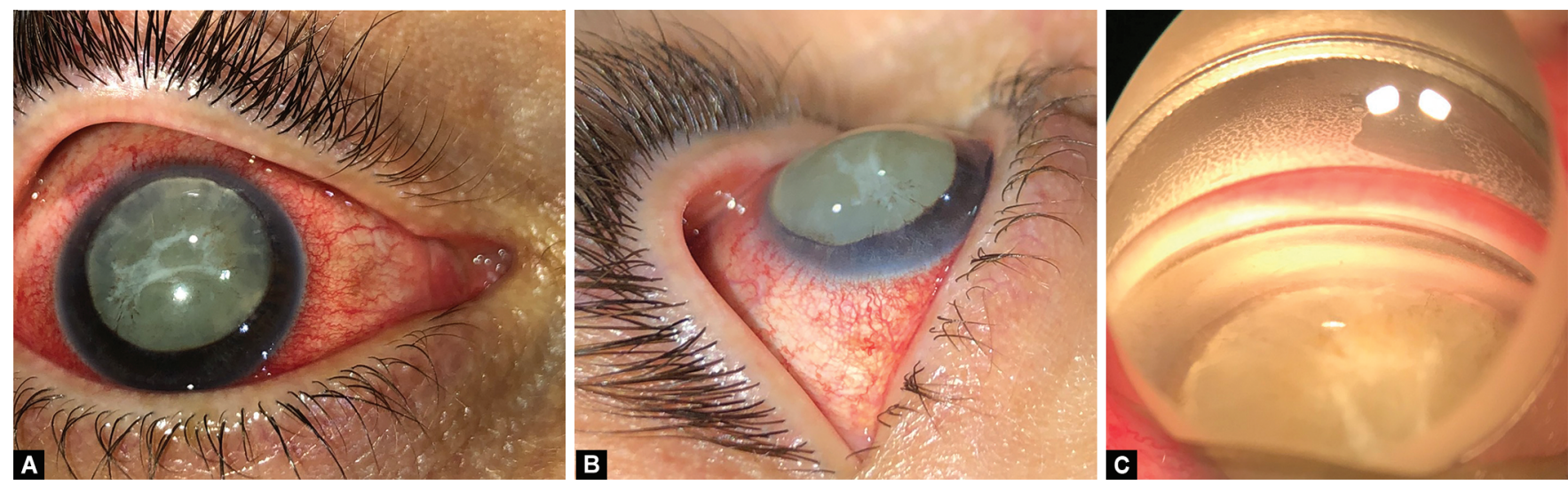

Figs $1 \mathrm{~A}$ to $\mathrm{C}$ : (A) Preoperative anterior segment photo of the right eye with a lens in the anterior chamber; (B) Side view of preoperative anterior segment examination of the right eye; (C) Gonioscopy of the right eye
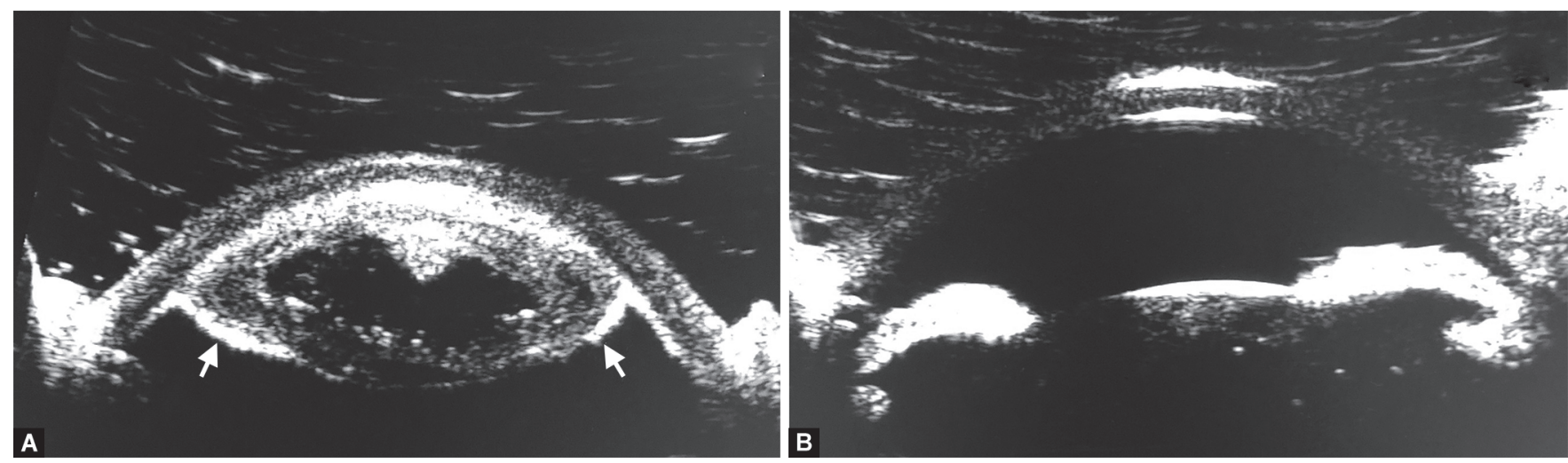

Figs 2A and B: (A) Ultrasound biomicroscopy image of the right eye. White arrows point to the iris located posterior to the lens; (B) Ultrasound biomicroscopy of left eye with normal anatomy 


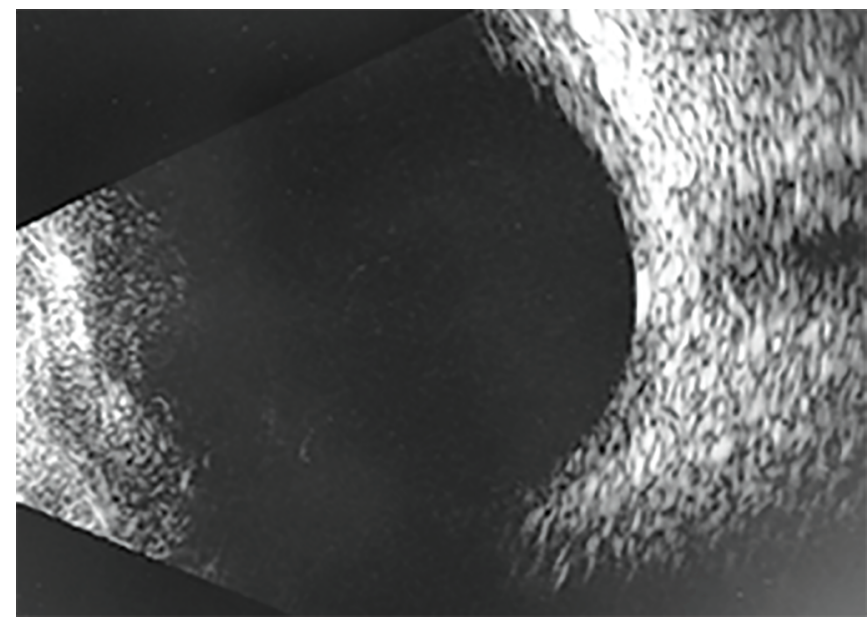

Fig. 3: B-scan ultrasound of right eye depicting no retinal detachment or tears

was measured and an $\mathrm{ACIOL}$ was placed and confirmed to be in a good position (Fig. 4). On postoperative day 1, the visual acuity was a response to bright light, with a formed anterior chamber and moderate IOP on palpation. On the month 1 visit, the home care attendant reported no more eye rubbing and appreciated an improvement in behavior and resolution of pain. The anterior chamber remained well-formed, with ACIOL in a good position.

\section{Discussion}

Both lens subluxation and self-induced trauma may produce unique and difficult scenarios, particularly in phakic patients. We report a unique case of complete subluxation of the natural lens into the anterior chamber, which required pars plana vitrectomy decompression, IZHV, and complex cataract surgery.

Anterior subluxation of the lens has been reported previously with different approaches resulting in successful treatment. These approaches include medical management, pars plana lensectomy and vitrectomy, and intracapsular lensectomy with anterior vitrectomy. ${ }^{7,11,12}$ Behndig described phacoemulsification and in-the-bag intraocular lens implantation in a case of anterior lens dislocation in spherophakia with intact zonules and shallow but formed anterior chamber. ${ }^{13}$ Kim and Ha described a case of intracapsular lens extraction combined with dry vitrectomy to allow for successful lens removal in a case with a flat anterior chamber and lens subluxation. ${ }^{8}$ In our case, we speculate her self-inflicted trauma caused zonular dehiscence and lead to the lens prolapsing into the anterior chamber as seen in Figure 1 and on UBM in Figure 2. The anterior subluxation induced malignant glaucoma and a flat anterior chamber which required a combination of techniques to successfully remove the cataract.

Given the flat anterior chamber and rapid improvement after IZHV, the mechanism of malignant glaucoma was felt to be multifactorial. We postulate the lens prolapsed into the anterior chamber and caused pupil seclusion and absolute pupillary block. The aqueous produced by the ciliary body was not able to travel into the anterior chamber due to the subluxated lens occupying the space. The buildup of aqueous in the posterior chamber caused the iris to be pushed anteriorly, creating a vicious cycle of malignant glaucoma.

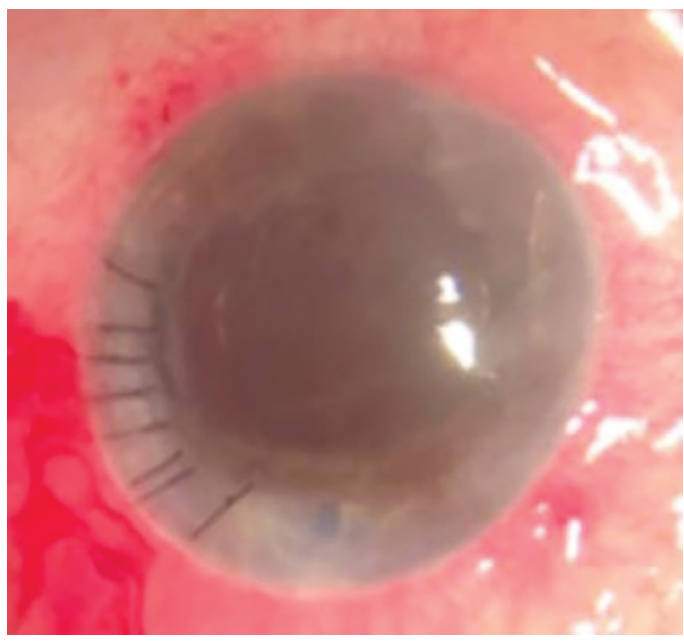

Fig. 4: End of case anterior segment photo of the right eye with $\mathrm{ACIOL}$ in position

Employing initial pars plana vitrectomy for debulking, followed by phacoemulsification of the lens, and finally, IZHV to treat malignant glaucoma has been described by both Sharma et al. and He et al. ${ }^{14,15}$ In these reports, IZHV was performed after cataract extraction. In our case, pars plana vitrectomy was first performed to debulk the vitreous and reduce posterior pressure, allowing for a limited reformation of the anterior chamber. Given the persistence of elevated pressure, IZHV was performed before cataract removal with deepening of the anterior chamber, which then allowed for successful cataract surgery.

The decision was made to proceed with phacoemulsification instead of manual small-incision cataract extraction because of the potential risk of expulsive choroidal hemorrhage. The elevated IOP and posterior pressure due to the anterior subluxation of the lens could have predisposed to developing expulsive choroidal hemorrhage, especially if there was a large incision. ${ }^{16}$

Finally, lens selection is an important consideration for all cataract surgeries. Options for our patient included scleral or iris fixation of the lens, ACIOL, or aphakia. In our case, the capsular bag was removed, thus excluding the option of a posterior chamber intraocular lens. A scleral fixated intraocular lens was deferred given the patients' history of self-induced trauma and the associated risk of lens tilt or dislocation, suture breakage. ${ }^{15,16}$ To maximize visual potential, we elected to place an ACIOL over aphakia. Though anterior chamber lenses have been associated with corneal endothelial cell loss, uveitis-glaucoma-hyphema syndrome, and other complications, these risks can be reduced and are comparable to posterior fixated lenses with proper measurement and placement. Each patient is unique and it is important to individualize lens selection to optimize the outcome for the patient being treated.

\section{Conclusion}

Malignant glaucoma can present in unique ways and the presence of a flat anterior chamber and increased posterior pressure can make surgery challenging. Imaging modalities like UBM can help plan surgical approach and initial pars plana vitrectomy and the creation of an IZHV can help relieve posterior pressure and facilitate cataract extraction. It is important to factor in patient-specific situations and goals while selecting an intraocular lens. 


\section{Clinical Significance}

Traumatic cataracts and malignant glaucoma can present in unique scenarios that may prove difficult to treat. Imaging can be helpful for surgical planning and certain techniques can facilitate these challenging surgeries and provide the optimal outcome for patients.

\section{ACKNOWLedgments}

Jean and David W. Wallace Family Foundation.

\section{References}

1. Quigley HA. Angle-closure glaucoma-simpler answers to complex mechanisms: LXVI Edward Jackson memorial lecture. Am J Ophthalmol 2009;148(5):657.e1-669.e1. DOI: 10.1016/j. ajo.2009.08.009.

2. Négrel $A D$, Thylefors B. The global impact of eye injuries. Ophthal Epidem 1998;5(3):143-169. DOI: 10.1076/opep.5.3.143.8364.

3. Jovanović M, Stefanović I. Spontaneous dislocation of a transparent lens to the anterior chamber - a case report. Srp Arh Celok Lek 2010;138(7-8):486-488. DOI: 10.2298/SARH1008486J.

4. Kawashima M, Kawakita T, Shimazaki J. Complete spontaneous crystalline lens dislocation into the anterior chamber with severe corneal endothelial cell loss. Cornea 2007;26(4):487-489. DOI: 10.1097/ ICO.0b013e3180303ae7.

5. Ritch R, Liebmann J, Tello C. A construct for understanding angleclosure glaucoma: the role of ultrasound biomicroscopy. Ophthalmol Clin N Amer 1995;8(2):281-293.

6. Wilde C, Morales MU, Kumudhan D, et al. Spontaneous onset pseudophakic malignant glaucoma secondary to zonular weakness and cilio-lenticular block. Oman J Ophthalmol. 2018;11(2):178-180. DOI: 10.4103/ojo.OJO_34_2016.

7. Garza-Leon M, De La Parra-Colín P. Medical treatment of crystalline lens dislocation into the anterior chamber in a patient with marfan syndrome. Int Ophthalmol 2012;32(6):585-587. DOI: 10.1007/s10792012-9592-7.

8. Kim YJ, Ha SJ. Intracapsular lens extraction for the treatment of pupillary block glaucoma associated with anterior subluxation of the crystalline lens. Case Rep Ophthalmol 2013;4(3):257-264. DOI: 10.1159/000356530.

9. Georgalas I, Ladas I, Papacostantinou D, et al. Management of crystalline lens dislocation into the anterior chamber in a victim of domestic violence. Clin Exp Optom 2012;95(1):113-115. DOI: 10.1111/j.1444-0938.2011.00637.x.

10. Lois N, Wong D, Groenewald C. New surgical approach in the management of pseudophakic malignant glaucoma. Ophthalmology 2001;108(4):780-783. DOI: 10.1016/S0161-6420(00)00642-4.

11. Peyman GA, Raichand M, Goldberg MF, et al. Management of subluxated and dislocated lenses with the vitrophage. $\mathrm{Br} J$ Ophthalmol 1979;63(11):771-778. DOI: 10.1136/bjo.63.11.771.

12. Choi D-Y, Kim J-G, Song B-J. Surgical management of crystalline lens dislocation into the anterior chamber with corneal touch and secondary glaucoma. J Cataract Refract Surg 2004;30(3):718-721. DOI: 10.1016/j.jcrs.2003.07.012.

13. Behndig A. Phacoemulsification in spherophakia with corneal touch J Cataract Refract Surg 2002;28(1):189-191. DOI: 10.1016/S08863350(01)00904-X.

14. Sharma A, Sii F, Shah P, et al. Vitrectomy-phacoemulsificationvitrectomy for the management of aqueous misdirection syndromes in phakic eyes. Ophthalmology 2006;113(11):1968-1973. DOI: 10.1016/j.ophtha.2006.04.031.

15. He F, Qian Z, Lu L, et al. Clinical efficacy of modified partial pars plana vitrectomy combined with phacoemulsification for malignant glaucoma. Eye 2016;30(8):1094-1100. DOI: 10.1038/eye.2016.106.

16. Ling $R$, Cole $M$, James $C$, et al. Suprachoroidal haemorrhage complicating cataract surgery in the UK: epidemiology, clinical features, management, and outcomes. Br J Ophthalmol 2004;88(4):478-480. DOI: 10.1136/bjo.2003.026138. 